\title{
Design and Analysis of a Three-Dimensional Discrete Memristive Chaotic System with Infinite Wide Parameter Range
}

Lilian Huang ( $\sim$ lilian_huang@163.com )

Harbin Engineering University

Jin Liu

Harbin Engineering University

Jianhong Xiang

Harbin Engineering University

Zefeng Zhang

Harbin Engineering University

\section{Research Article}

Keywords: Discrete Memristor, Discrete Chaotic Map, Infinitely Wide Parameter Range, Super Multiple Stability

Posted Date: November 29th, 2021

DOI: https://doi.org/10.21203/rs.3.rs-1109068/v1

License: (9) This work is licensed under a Creative Commons Attribution 4.0 International License. Read Full License 


\title{
Design and analysis of a three-dimensional discrete memristive chaotic system with infinite wide parameter range
}

\author{
Lilian Huang · Jin Liu · Jianhong Xiang · Zefeng Zhang
}

Received: date / Accepted: date

\begin{abstract}
In this paper, a new discrete memristive chaotic system with infinitely wide parameter range is designed. Firstly, a discrete memristor based on a triangular wave function is constructed. The memristor conforms to the definition of generalized memristor, and a new three-dimensional memristive chaotic system is designed based on it. Numerical simulations show that it can generate chaotic sequences with high complexity.Otherwise, an improved perturbation method is proposed to estimate the output sequence of the differential system. At the same time, it is proved mathematically that the new system can always be in chaotic or hyperchaotic state with infinitely wide parameter range under certain conditions. By observing the Lyapunov exponent spectrum and the phase diagram, it is found as the absolute value of the parameter increases, the output range and ergodicity of the new system are also enhanced, and the new system has super multistability. This paper analyzes the mechanism of the discrete memristive chaotic system generating infinitely coexisting attractors, puts forward a method to make ordinary chaotic systems easier to obtain super multistability, and verifies it. The results show it is effective. Finally, the DSP hardware platform is used to implement the new system, which proves the physical existence and realizability of the system.
\end{abstract}

Keywords Discrete Memristor - Discrete Chaotic Map · Infinitely Wide Parameter Range · Super Multiple Stability

L. Huang · J. Liu · J. Xiang $(\bowtie) \cdot$ Z. Zhang

College of Information and Communication Engineering, Harbin Engineering University Harbin 150001, China

MIIT Key Laboratory of Advanced Marine Communication and Information Technology, Harbin 150001, China

E-mail: xiangjianhong@hrbeu.edu.cn

\section{Introduction}

As a new type of nonlinear components, memristors have widely used in artificial neural networks [1,2], logic operations[3], non-volatile memory[4], chaotic oscillator circuit design $[5,6,7,8]$ and other fields. Compared with typical chaotic circuits, the chaotic signal generated by the memristive chaotic system has better dynamic behavior, and it is easier to design chaotic circuits with super multi-stability properties $[9,10]$. Therefore, it is of great significance to study and design the memristive chaotic circuits with superior performance.

Compared with the continuous chaotic system, discrete chaotic map has the advantages of faster operation speed and higher bit rate. Therefore, it is more suitable for constructing pseudo-random sequences. Common one-dimensional(1D) discrete chaotic maps, such as Logistic mapping, Tent map, Sine map, and Gauss map, usually have the disadvantages of simple structure, small chaotic area, and uneven signal distribution[11]. Otherwise, high-dimensional chaotic map has a larger key space and better chaotic performance, and are more likely to produce hyperchaotic attractors[12, 13]. Besides, the high-dimensional discrete hyperchaotic systems with super multi-stability have richer dynamic behaviors[14], which can be well applied in practice.

Many existing chaotic systems have small and discontinuous chaotic intervals, which means that there have many periodic windows in the chaotic intervals of these systems. Small disturbances to their control parameters can easily cause the parameters to fall into the periodic window, thus making the system lose chaotic behavior. However, compared with a system with narrow range, the chaotic system with wide range or even an infinite range of parameters has stronger anti-interference, and it tends to be more flexible, reliable, and sta- 
ble in the practical engineering applications. Therefore, it is necessary to study and design the chaotic systems with wide parameter range.

At present, most continuous chaotic systems with wide parameter range are studied. In 2013, Liu[15] proposed a $3 \mathrm{D}$ chaotic system with a parameter range of $[-100,100]$. In $2019, \mathrm{Xu}[16]$ proposed a $3 \mathrm{D}$ continuous chaotic system with a parameter range of $[0,107]$. Zhou[17] proposed a hyperchaotic system with a parameter range of $[1,107]$ and implemented it with FPGA. In 2020, Xu, Zhong[18] proposed a large-scale chaotic system with coexisting attractors. In 2021, Zhang[19] proposed a $5 \mathrm{D}$ conservative hyperchaotic system with a parameter range of $[0,1500]$. However, discrete chaotic systems with wide or even infinite wide parameter ranges are rarely studied.

Based on above-mentioned research, this paper designs a new discrete memristive chaotic system with infinite wide parameter range. Under suitable conditions, this new system can keep chaotic state or a hyperchaotic state within an infinitely wide parameter range. By comparing the Lyapunov exponent and phase diagram, it can be found that the dynamic behavior of the system will be strengthened with the increase of the absolute value of the control parameters. Meanwhile, the new system also has super multi-stability properties. The attractors of the system, including periodic, chaotic and hyperchaotic attractors, will be changed by the initial value. By analyzing this phenomenon, this paper summarizes the method of transforming the common discrete chaotic map to obtain super multistability properties. When applying it to an ordinary $3 \mathrm{D}$ discrete chaotic map, the result proves that the method is feasible. Finally, the new system is accomplished by using the DSP hardware platform.

The rest of this paper is organized as follows. Section 2 constructs a discrete memristor and proves that it meets the definition of generalized memristor. Section 3 presents a new 3D discrete memristive chaotic system, and analyses the influence of the control parameters, which mathematically proved that the new system has a chaotic region with an infinitely wide parameter range. Section 4 analyses and verifies the mechanism of the discrete memristive chaotic system generating inginitely coexisting attractors. Section 5 implements the memristive chaotic system in a hardware platform. Finally, Section 6 draws a conclusion of this paper.

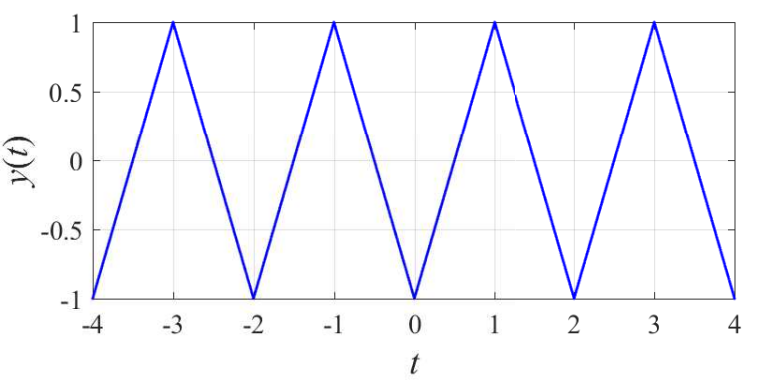

Fig. 1: Triangle wave waveform

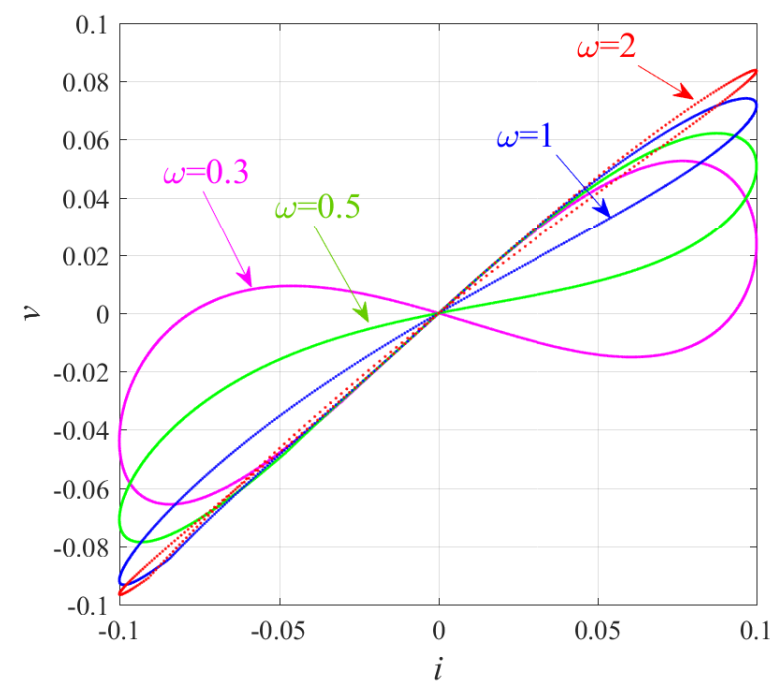

Fig. 2: The volt-ampere characteristic curve of the memristor during $\omega$ transformation

\section{Construction of a new discrete memristor}

First, we will construct a new discrete memristor and the memristive function is shown in Equation (2-1).

$M\left(q_{n}\right)=\operatorname{tri}\left(q_{n}\right)$

The memristive function is a triangular wave function with a period of 2 and an amplitude of 1 , and $q_{n}$ is the input of the triangular wave function.

Among them, $q_{n}$ is the value of the electric charge at the $n$th iteration, and the waveform of the triangular wave function $y(t)=\operatorname{tri}(t)$ is shown in the Fig.1.

In order to verify the properties of the discrete memristor, set the input current as $i_{n}=A \sin (\omega n)$ and the input voltage as $v_{n}=M\left(q_{n}\right) i_{n}$. Referring to the literature of Li et al. [14], define $q_{n+1}=q_{n}+i_{n}$ and fix $A=0.1, q_{0}=1$, and the tight hysteresis loop of the memristor at different frequencies is shown in the Fig.2. 


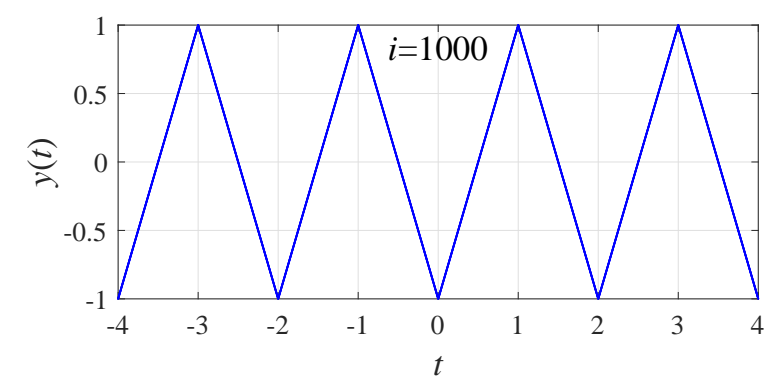

Fig. 3: Fourier series expansion of the triangular wave function

It can be seen from the Fig.2 that the memristor model conforms to the relevant definition of memristor[20,21].

\section{New three-dimensional discrete memristive chaotic system}

Define the difference equations of a 3D discrete system as:

$\left\{\begin{array}{l}x_{n+1}=0.2 \sin \left(\pi x_{n}\right)+k M\left(q_{n}\right) y_{n} \\ y_{n+1}=a \cos \left(\pi x_{n}\right)+\cos \left(\pi y_{n}\right) \\ q_{n+1}=q_{n}+0.6 \sin \left(\pi q_{n}\right)+0.2 \sin \left(\pi y_{n}\right)\end{array}\right.$

Where $k$ and $a$ are the control parameters of the system, $x_{n}, y_{n}$, and $q_{n}$ are the state variables of the system, and $M\left(q_{n}\right)$ is the memristive function as shown in Equation (2-1).

\subsection{The influence of control parameters}

We expands the triangular wave function into Fourier series to approximate it, as shown in Formula (3-2).

$y(t)=\sum_{i=1}^{\infty} \frac{4}{(i \pi)^{2}}[\cos (i \pi)-1] \cos (i \pi t)$

To obtain a better approximation effect, set $i=1000$, as shown in the Fig.3.

When the initial value is $[1,1,0.8]$, let the system parameters and gradually increase. We get the 3D maximum Lyapunov exponent $\left(L E_{\max }\right)$ and dynamic map as shown in the Fig.4.

It can be seen from the Fig. 4 that the system only has a partial periodic window around $k=0$ or $a=$ 0 . On the whole, as the absolute value of the system parameter increases, the period windows around $k=0$ or $a=0$ tend to be narrower. in addition, the $L E_{\max }$ is greater than 0 in other regions, which means that the system is always in a chaotic or hyperchaotic state
Table 1: Several different values of system parameters and the corresponding values of Lyapunov exponents and states

\begin{tabular}{ccc}
\hline \multicolumn{1}{c}{$k, a$} & LEs & system state \\
\hline $0.01,0.01$ & $-0.131,-0.461,-2.299$ & Cycle 1 \\
$0.04,0.4$ & $-0.414,-0.603,-1.303$ & Cycle 4 \\
$0.35,0.14$ & $0.535,-0.436,-0.884$ & Chaotic state \\
$0.8,3$ & $0.940,0.602,-0.0 .45$ & Hyperchaotic state \\
10,10 & $2.533,2.292,1.403$ & Hyperchaotic state \\
$10^{10}, 10^{10}$ & $23.230,23.210,22.080$ & Hyperchaotic state \\
\hline
\end{tabular}

in these regions without state fading phenomenon, and $L E_{\text {max }}$ increases with the increase of the absolute value of $k$ and $a$, indicating that the chaotic characteristics are also stronger.

Let the initial value remains unchanged, and set the control parameters $k$ and $a$ as several typical values, as shown in Table 1.

The corresponding phase diagram is shown in the Fig.5.

It can be seen from Table 1 and Fig.5 the new system exhibits rich dynamic behaviors with different parameters. When $k$ and $a$ gradually increase, the overall Lyapunov exponents also increases, and the system state changes from a periodic state to chaotic state and then enters a hyperchaotic state. When $k$ and $a$ are both $10^{10}$, the three Lyapunov exponents can all reach more than 20, indicating that the system has excellent chaotic characteristics. At the same time, it can be seen from the phase diagram that the attractors at this time presents a regular cylinder, in which the iteration sequence of the system is evenly distributed and the ergodicity is stronger.

The initial value is set to $\left[1,1, q_{0}\right]$, and fix $a=$ 10. When $k$ is gradually increased, describe the Lyapunov exponent spectrum and the bifurcation diagram as shown in the Fig.6 and Fig.7.

It can be seen from the Fig.6, when the initial value is set as $[1,1,0.8]$, and $a=10$, the new system only has a very narrow period window in some areas near $k=0$. In the remaining regions, with the increase of $k$, the system state quickly enters the chaotic state and then to the hyperchaotic state.

As can be seen from the bifurcation diagram of the state variable $q_{n}$, different $q_{0}$ produces different corresponding bifurcation diagrams, which proves the existence of the system's coexistence attractor. This phenomenon will be further discussed in the following sections.

It can be seen from the Fig. 7 when other conditions remain unchanged, and $k$ is gradually increased from 0 to $5 \times 10^{7}$, the three Lyapunov exponents of the system 


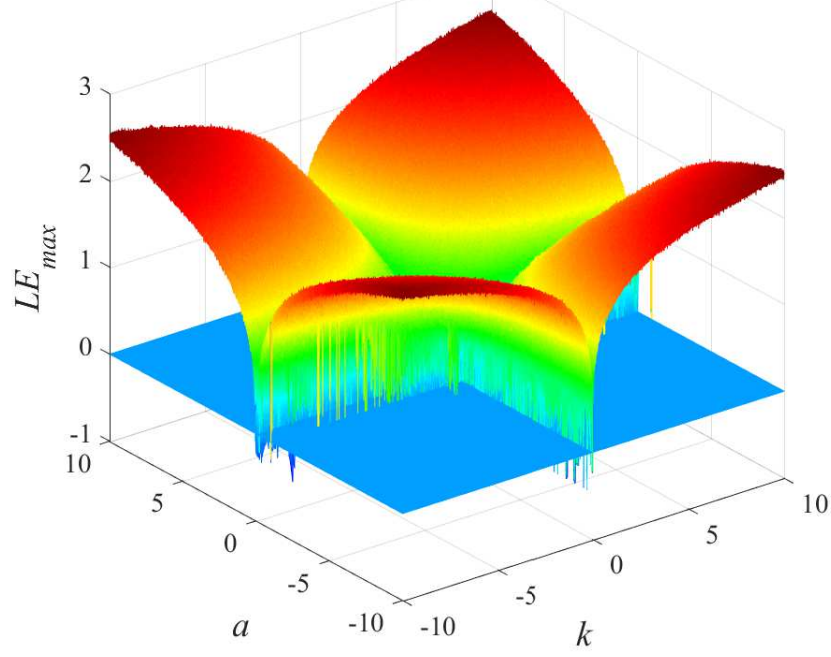

(a) The maximum Lyapunov exponent of $k, a \in[-10,10]$

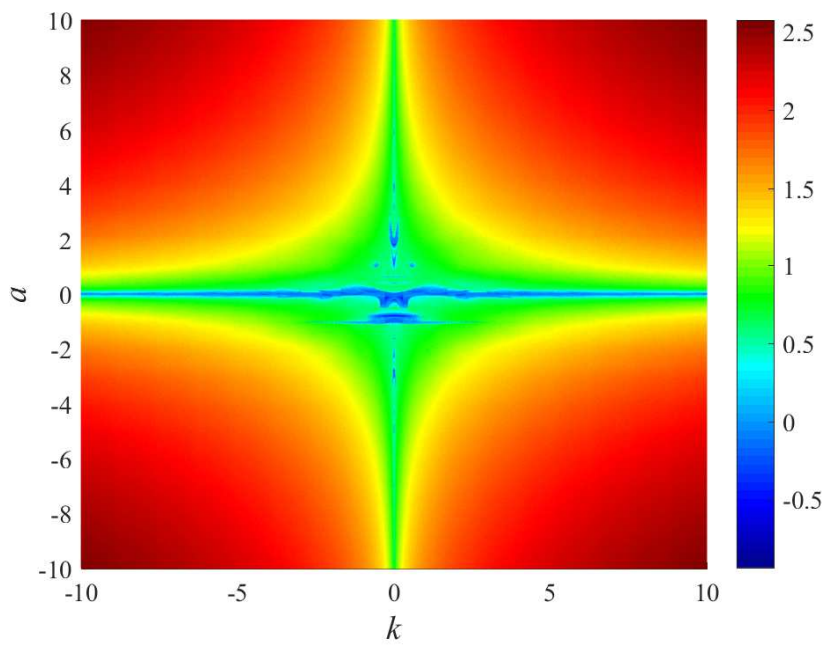

(b) Dynamic map of $k, a \in[-10,10]$

Fig. 4: The maximum Lyapunov exponent of the new system and its corresponding dynamic map

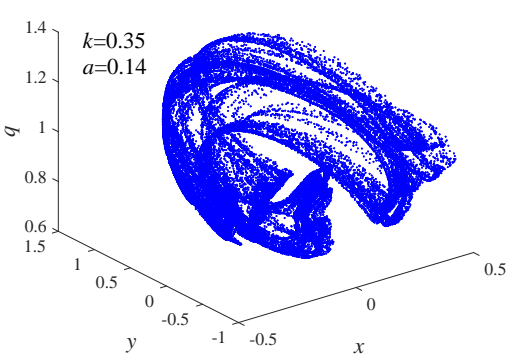

(a) Chaotic state

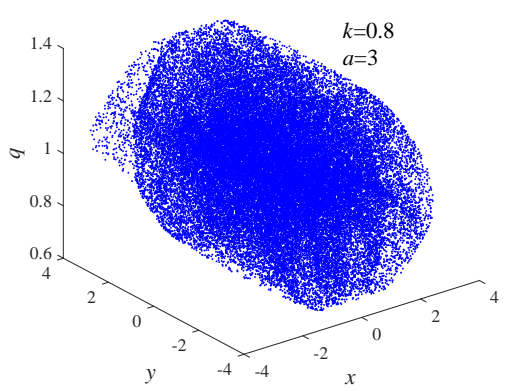

(d) Hyperchaotic state

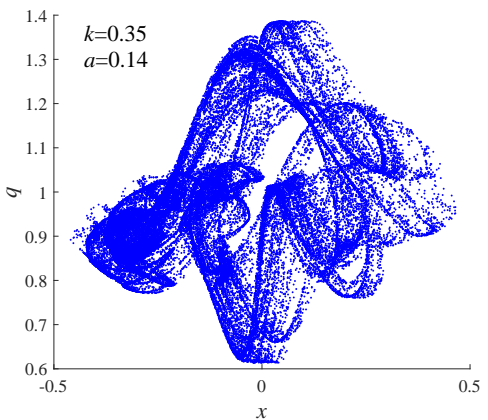

(b) $x-q$ plane phase diagram

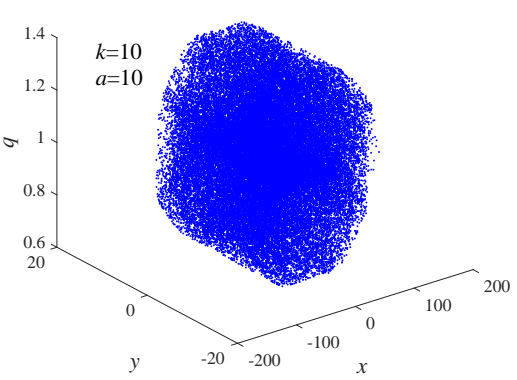

(e) Hyperchaotic state

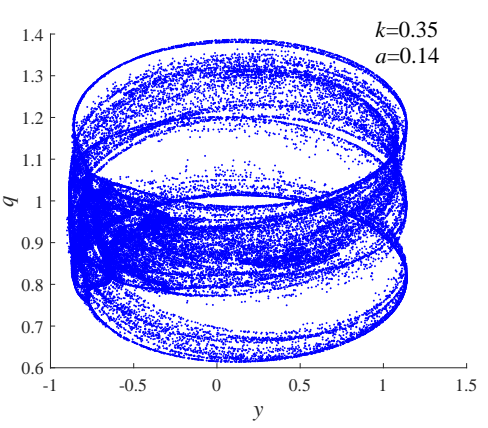

(c) $y-q$ plane phase diagram

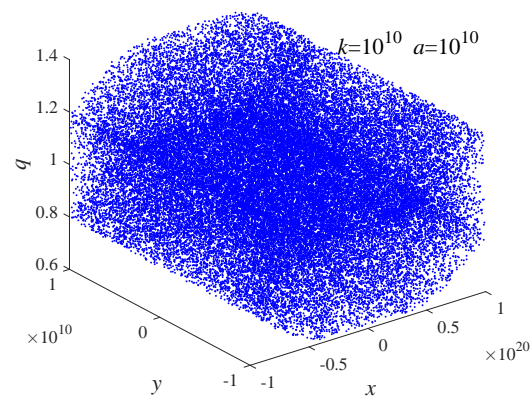

(f) Hyperchaotic state

Fig. 5: Phase diagrams of several different states of the new system 

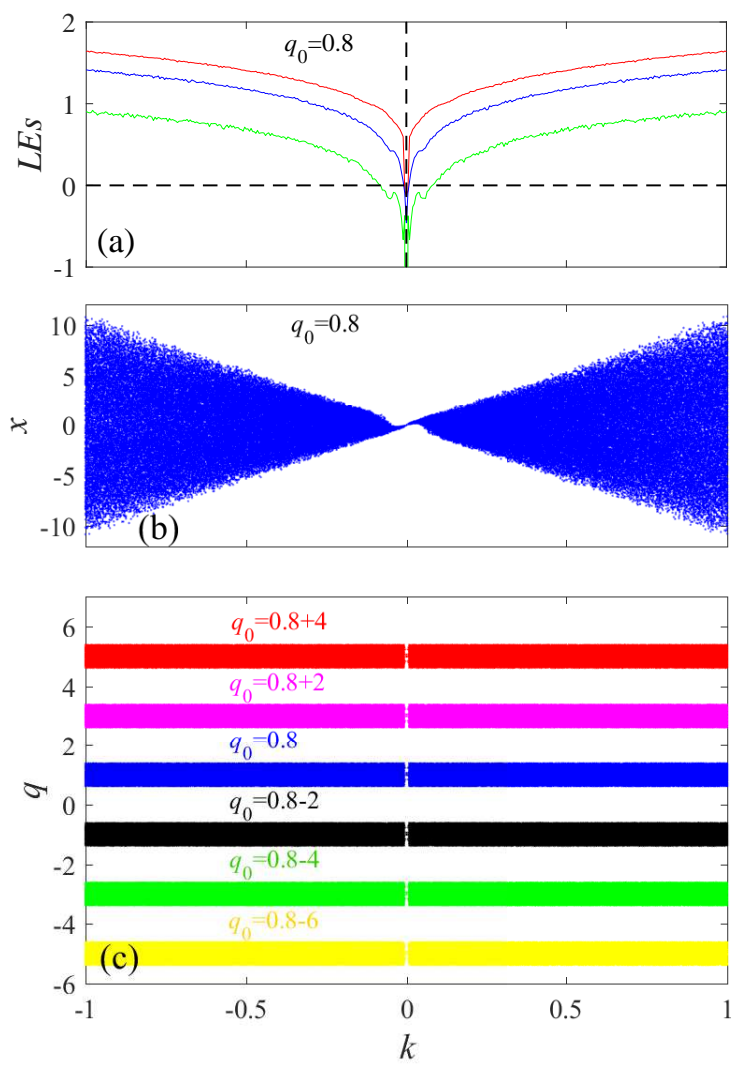

Fig. 6: Lyapunov exponent spectrum of the new system and its corresponding bifurcation diagram (a) Lyapunov exponent spectrum of the new system; (b) bifurcation diagram of state variable $x_{n} ;$ (c) bifurcation diagram of state variable $q_{n}$

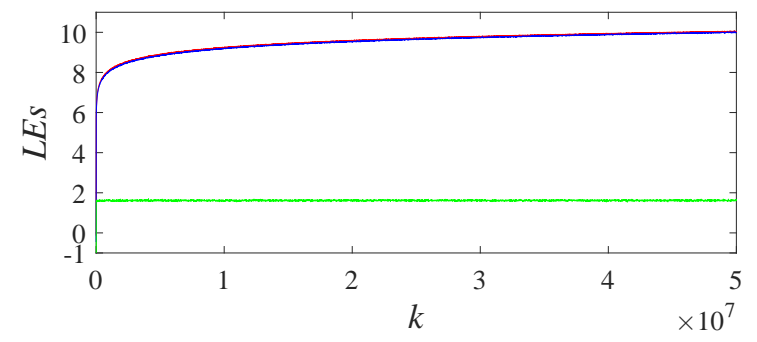

Fig. 7: Lyapunov exponent with parameter $k \in[0.5 \times$ $\left.10^{7}\right]$

are still above 0. Although the first and second Lyapunov exponents generally increase with the increase of parameters and gradually approach each other, the increasing speed is getting slower, while the smallest Lyapunov exponent remains stable around 1.6. Besides, after the system enters the hyperchaotic state, the state will remain unchanged without any periodic window.

\subsection{Chaos zone with infinite parameter range}

To show that the system (3-1) is chaotic or hyperchaotic within an infinitely wide range of parameters, it only needs to meet the Following two criteria[22].

(1)The orbit is globally bounded;

(2)The Lyapunov exponents are positive.

A strict mathematical proof will be given below.

\subsubsection{Proof of global boundedness}

Obviously, no matter what the initial value is, when $k, a \in \mathrm{R}$ ( $\mathrm{R}$ is the real number domain), $x_{n+1}$ and $y_{n+1}$ in the difference equation are always bounded functions. Therefore, $x_{n+1}$ and $y_{n+1}$ are globally bounded.

In order to prove the global boundedness of $q_{n}$, the function $f(x)$ is defined as follows:

$f(x)=x+0.6 \sin (\pi x)-0.2, x \in[-1.4,2]$

The function $f(x)$ is continuously differentiable in the domain $x \in[-1.4,2]$, and its maximum and minimum values are taken at the endpoints or poles. It can be obtained by calculation:

$$
\left\{\begin{array}{l}
f(x)_{\max }=f(2)=1.8 \\
f(x)_{\min } \approx f(-0.678) \approx-1.387
\end{array}\right.
$$

That is, the equation $f(x)$ satisfies:

$$
-1.4 \leq x \leq 2,-1.4 \leq f(x) \leq 2
$$

Construct the difference equation:

$u_{n+1}=u_{n}+0.6 \sin \left(\pi u_{n}\right)-0.2$

Let the difference equation (3-5) iterate with different initial values $u_{0}^{i}$ until the $n$th iteration value is $u_{n}^{i}$, then the output matrix is as follows:

$\left[\begin{array}{cccc}u_{0}^{1} & u_{1}^{1} & \cdots & u_{n}^{1} \\ u_{0}^{2} & u_{1}^{2} & \cdots & u_{n}^{2} \\ \vdots & \vdots & \ddots & \vdots \\ u_{0}^{i} & u_{1}^{i} & \cdots & u_{n}^{i}\end{array}\right]$

Let $u_{0}^{1} \in[0,2]$, it is easy to prove is globally bounded, and its range is $-1.4 \leq u_{n}^{1} \leq 2$. The proof process is as follows:

(1) When $n=0$, it is obvious that,

$-1.4 \leq u_{0}^{1} \leq 2$

the proposition is true;

(2)Suppose when $n=m, m \in \mathrm{N}^{*}\left(\mathrm{~N}^{*}\right.$ is a positive integer),

$-1.4 \leq u_{m}^{1} \leq 2$ 
the proposition is true;

(3)When $n=m+1$, it can be known from the equation (3-4) and relational equation (3-5), when

$-1.4 \leq u_{m}^{1} \leq 2$

then

$-1.4 \leq u_{m}^{1}+0.6 \sin \left(\pi u_{m}^{1}\right)-0.2 \leq 2$

where

$-1.4 \leq u_{m+1}^{1} \leq 2$

the proposition is true.

When $u_{0}^{2} \in[2,4]$, suppose that each value point of $u_{0}^{2}$ corresponds to the value point of $u_{0}^{1}$ in a one-to-one correspondence with period 2 , then $u_{1}^{2}$ is:

$$
\begin{aligned}
u_{1}^{2} & =u_{0}^{2}+\sin \left(\pi u_{0}^{2}\right)-0.2 \\
& =\left(u_{0}^{1}+2\right)+\sin \left[\pi\left(u_{0}^{1}+2\right)\right]-0.2 \\
& =u_{1}^{1}+2
\end{aligned}
$$

Substitute it into the equation (3-3) for iteration, the output sequence of $u_{n}^{2}$ is obtained as follows:

$$
\left[u_{0}^{2}, u_{1}^{2}, \cdots, u_{n}^{2}\right]=\left[u_{0}^{1}+2, u_{1}^{1}+2, \cdots, u_{n}^{1}+2\right]
$$

where

$-1.4+2 \leq u_{n}^{2} \leq 2+2$

Similarly, for different initial values, is always globally bounded.

Again, construct the difference equation:

$w_{n+1}=w_{n}+0.6 \sin \left(\pi w_{n}\right)+0.2$

It can also prove that $w_{n}$ is globally bounded under different initial values. The proof method is as above.

And because

$$
\begin{aligned}
& q_{n}+0.6 \sin \left(\pi q_{n}\right)-0.2 \\
& \leq q_{n}+0.6 \sin \left(\pi q_{n}\right)-0.2 \sin \left(\pi y_{n}\right) \\
& \leq q_{n}+0.6 \sin \left(\pi q_{n}\right)+0.2
\end{aligned}
$$

From equations (3-5) and (3-6), we know that $q_{n+1}$ is globally bounded.

\subsubsection{Proof of positive Lyapunov exponent}

In order to prove that the new system has an infinitely wide parameter range of the positive Lyapunov exponent, when $k$ and $a$ are directly used as unknown variables to obtain the analytical solution of the system's Lyapunov exponent, a large number of iterative operations on the system (3-1) are needed to obtain its output sequence. This method has a huge amount of calculation and almost impossible to complete. Therefore, this paper proposes an improved perturbation method, which can estimate the output sequence of the difference equation without substituting specific parameter values. This method avoids a large number of iterative operations on substituting equations, and can obtain the output sequence of the equation with little loss of accuracy. It should be noted that this method is to estimate the output sequence of the system, which requires the Lyapunov exponent, and the traditional Lyapunov exponent calculation should be carried out on the basis of the output sequence.

The basic idea of the perturbation method is that if a system contains a parameter $\gamma$ which is difficult to locate accurately, the system can be degenerated to $\gamma_{0}=0$ first, and in the case of solving the motion expression of the system $\gamma_{0}=0$, the system is regarded as a disturbed system formed by a small perturbation at the parameter $\gamma_{0}=0$, that is, $\gamma=\gamma_{0}+\varepsilon$ at this time. Based on this, the solution of the system under the disturbance can be found, so as to simplify the mathematical processing difficulty.The improved perturbation method will be introduced below.

Suppose the expression of the difference equation is as follows:

$\left\{\begin{array}{c}x_{1}(n+1)=f_{1}\left(u, x_{1}(n), x_{2}(n), \cdots, x_{j}(n)\right) \\ x_{2}(n+1)=f_{2}\left(u, x_{1}(n), x_{2}(n), \cdots, x_{j}(n)\right) \\ \vdots \\ x_{j}(n+1)=f_{j}\left(u, x_{1}(n), x_{2}(n), \cdots, x_{j}(n)\right)\end{array}\right.$

$x_{j}(n)$ is the $n$th iteration value of the th state variable, and $u$ is the control parameter, $f: \mathrm{R}^{\mathrm{j}} \rightarrow \mathrm{R}^{\mathrm{j}}$.

Now take $u=u_{0}$ as the base point, and set the initial value $\left[x_{1}(0), x_{2}(0), \cdots, x_{j}(0)\right]^{T}$. At this time, there is no unknown quantity in the system, then the iterative output matrix of the system (3-7) is:

$H=\left[\begin{array}{cccc}x_{1}(0) & x_{1}(1) & \cdots & x_{1}(n) \\ x_{2}(0) & x_{2}(1) & \cdots & x_{2}(n) \\ \vdots & \vdots & \ddots & \vdots \\ x_{j}(0) & x_{j}(1) & \cdots & x_{j}(n)\end{array}\right]$ 
Table 2: Lyapunov exponents of two different methods

\begin{tabular}{clll}
\hline$k$ & Traditional method & \multicolumn{2}{l}{ Improved perturbation method } \\
\hline $10^{2}$ & $3.574,3.380,1.670$ & $i=90$ & $3.570,3.363,1.621$ \\
$10^{3}$ & $4.684,4.558,1.601$ & $i=990$ & $4.683,4.557,1.630$ \\
$10^{4}$ & $5.817,5.711,1.610$ & $i=9990$ & $5.808,5.709,1.628$ \\
$10^{5}$ & $6.961,6.874,1.621$ & $i=99990$ & $6.958,6.877,1.628$ \\
$10^{6}$ & $8.102,8.035,1.619$ & $i=999990$ & $8.100,8.032,1.629$ \\
\hline
\end{tabular}

Set $u_{1}=u_{0}+\Delta u, \Delta u$ is a small increment, and the output matrix is:

$H_{\Delta}=\left[\begin{array}{cccc}x_{1}(0) & x_{1}^{\Delta}(1) & \cdots & x_{1}^{\Delta}(n) \\ x_{2}(0) & x_{2}^{\Delta}(1) & \cdots & x_{2}^{\Delta}(n) \\ \vdots & \vdots & \ddots & \vdots \\ x_{j}(0) & x_{j}^{\Delta}(1) & \cdots & x_{j}^{\Delta}(n)\end{array}\right]$

On the basis of the output matrix , the improved perturbation method makes $x_{j}^{1}(n)\left(n \in \mathrm{N}^{*}\right)$ perform Taylor series expansion near the base point $u=u_{0}$, namely:

$x_{j}^{1}(n)=f_{j}+\left(u_{1}-u_{0}\right) f_{j}^{\prime}+\cdots+\frac{\left(u_{1}-u_{0}\right)^{n} f_{j}^{(n)}}{n !}+R_{n}$

According to the requirements of calculation accuracy, select a suitable position for truncation, and then substitute $x_{j}^{1}(n)$ back into $H_{\Delta}$ to obtain the output matrix of the system at the parameter $u_{1}=u_{0}+\Delta u$ is obtained.

For the system (3-1), as $k$ only exists in the expression $x_{n+1}$, and $k$ is a first-order term, $x_{n+1}=f_{1}\left(k, x_{n}\right.$, $\left.y_{n}, q_{n}\right)$ can be regarded as a first-order function of the parameter $k$, and the influence of parameter $k$ on $y_{n+1}$ and $q_{n+1}$ is ignored here. Therefore, when Taylor series expansion is carried out, the perturbation range can get rid of the limitation that only a small amount can be taken.

Set the initial value as $[1,1,0.8]$, fix $a=10$, and set $k_{0}=10$ as the base point. Then, when $k_{i}=k_{0}+i, i \in$ $\mathrm{N}^{*}$, and $i$ is the perturbation increment, the system output matrix is:

$$
\left[\begin{array}{cccc}
x_{0} & x_{1}+i \operatorname{tri}\left(q_{1}\right) y_{1} & \cdots & x_{n}+i \operatorname{tri}\left(q_{n}\right) y_{n} \\
y_{0} & y_{1} & \cdots & y_{n} \\
q_{0} & q_{1} & \cdots & q_{n}
\end{array}\right]
$$

Let $a=10$, Table 2 makes a comparison of the Lyapunov exponents solved by two different methods.

It can be seen that the improved perturbation method calculates the output sequence of the system with different by using the perturbation increment, and then solves the Lyapunov exponent. Compared with traditional methods, the results are similar.
Next, this paper will use mathematical induction to prove the positive Lyapunov exponent of the infinitely wide parameter range of the system (3-1), and prove that $L E_{1}$ gradually increases with the increase of the parameter $k$.

First, solve Lyapunov exponent by definition method as follows:

$\varepsilon e^{n \lambda}=\left|F^{n}\left(x_{0}+\varepsilon\right)-F^{n}\left(x_{0}\right)\right|$

Take the limit $\varepsilon \rightarrow 0, n \rightarrow \infty$, then

$\lambda=\lim _{\substack{n \rightarrow \infty \\ \varepsilon \rightarrow 0}} \frac{1}{n} \ln \left|\frac{F^{n}\left(x_{0}+\varepsilon\right)-F^{n}\left(x_{0}\right)}{\varepsilon}\right|$

$\lambda$ is the Lyapunov exponent of the system.

Set $a=10, k_{0}=10$, and the Lyapunov exponents obtained by QR orthogonal method are $L E_{1}^{0}=2.533$, $L E_{2}^{0}=2.292$ and $L E_{3}^{0}=1.403$. When using the definition method to solve the Lyapunov exponent, set the two initial values $\left[x_{0}, y_{0}, q_{0}\right]$ and $\left[x_{0}+\varepsilon, y_{0}, q_{0}\right]$, with small differences firstly, and the output matrices respectively are:

$\left[\begin{array}{llll}x_{0} & x_{1} & \cdots & x_{n} \\ y_{0} & y_{1} & \cdots & y_{n} \\ q_{0} & q_{1} & \cdots & q_{n}\end{array}\right]$

$\left[\begin{array}{cccc}x_{0}+\varepsilon & x_{11} & \cdots & x_{n n} \\ y_{0} & y_{11} & \cdots & y_{n n} \\ q_{0} & q_{11} & \cdots & q_{n n}\end{array}\right]$

From equation (3-8) and $L E_{1}^{0}=2.533$, we can obtain:

$L E_{1}^{0}=\lim _{\substack{n \rightarrow \infty \\ \varepsilon \rightarrow 0}} \frac{1}{n} \ln \left|\frac{x_{n n}-x_{n}}{\varepsilon}\right|>0$

Set

$\frac{x_{n n}-x_{n}}{\varepsilon}=S_{1}$

Then we can obtain:

$\left|S_{1}\right|>1$

(1)When $i=1$, that is, $k_{1}=k_{0}+1=11$, it is easy to calculate that the specific Lyapunov exponents of the system (3-1) are $L E_{1}^{1}=2.553, L E_{2}^{1}=2.315$ and $L E_{3}^{1}=1.4909$. In this case, the Lyapunov exponents of the system are all positive, and $L E_{1}^{1}>L E_{1}^{0}$.

When using the definition method to solve the Lyapunov exponent, the output matrices of the system at different initial values are first obtained as follows:

$\left[\begin{array}{cccc}x_{0}^{1} & x_{1}^{1} & \cdots & x_{n}^{1} \\ y_{0}^{1} & y_{1}^{1} & \cdots & y_{n}^{1} \\ q_{0}^{1} & q_{1}^{1} & \cdots & q_{n}^{1}\end{array}\right]$
$=\left[\begin{array}{cccc}x_{0} & x_{1}+\operatorname{tri}\left(q_{1}\right) y_{1} & \cdots & x_{n}+\operatorname{tri}\left(q_{n}\right) y_{n} \\ y_{0} & y_{1} & \cdots & y_{n} \\ q_{0} & q_{1} & \cdots & q_{n}\end{array}\right]$




$$
\begin{gathered}
{\left[\begin{array}{cccc}
x_{0}^{1}+\varepsilon & x_{11}^{1} & \cdots & x_{n n}^{1} \\
y_{0}^{1} & y_{11}^{1} & \cdots & y_{n n}^{1} \\
q_{0}^{1} & q_{11}^{1} & \cdots & q_{n n}^{1}
\end{array}\right]} \\
=\left[\begin{array}{cccc}
x_{0}+\varepsilon & x_{1}+\operatorname{tri}\left(q_{1}\right) y_{1} & \cdots & x_{n}+\operatorname{tri}\left(q_{n}\right) y_{n} \\
y_{0} & y_{1} & \cdots & y_{n} \\
q_{0} & q_{1} & \cdots & q_{n}
\end{array}\right]
\end{gathered}
$$

Then the definition method of the first Lyapunov exponent is solved as:

$L E_{1}^{1}=\lim _{\substack{n \rightarrow \infty \\ \varepsilon \rightarrow 0}} \frac{1}{n} \ln \left|S_{1}+\frac{\operatorname{tri}\left(q_{n n}\right) y_{n n}-\operatorname{tri}\left(q_{n}\right) y_{n}}{\varepsilon}\right|>0$

Set

$\frac{\operatorname{tri}\left(q_{n n}\right) y_{n n}-\operatorname{tri}\left(q_{n}\right) y_{n}}{\varepsilon}=S_{2}$

Then we can obtain:

$\left|S_{1}+S_{2}\right|>1$

$\left|S_{1}+S_{2}\right|-\left|S_{1}\right|>0$

The proposition is true.

(2)Suppose the proposition is true when $i=m$, that is, $k_{m}=k_{0}+m$. At this time, the $L E_{1}^{m}>0$, and $L E_{1}^{m} \geq L E_{1}^{m-1}$. The output matrix and the first Lyapunov exponent are as follows:

$$
\begin{aligned}
& {\left[\begin{array}{cccc}
x_{0} & x_{1}+m \operatorname{tri}\left(q_{1}\right) y_{1} & \cdots & x_{n}+m \operatorname{tri}\left(q_{n}\right) y_{n} \\
y_{0} & y_{1} & \cdots & y_{n} \\
q_{0} & q_{1} & \cdots & q_{n}
\end{array}\right]} \\
& {\left[\begin{array}{ccc}
x_{0}+\varepsilon & \cdots & x_{n n}+m \operatorname{tri}\left(q_{n n}\right) y_{n n} \\
y_{0} & \cdots & y_{n n} \\
q_{0} & \cdots & q_{n n}
\end{array}\right]}
\end{aligned}
$$

$L E_{1}^{m}=\lim _{\substack{n \rightarrow \infty \\ \varepsilon \rightarrow 0}} \frac{1}{n} \ln \left|S_{1}+m S_{2}\right|>0$

where

$\left|S_{1}+m S_{2}\right|>1$

$\left|S_{1}+m S_{2}\right|-\left|S_{1}+(m+1) S_{2}\right|>0$

(3) When $i=m+1$, the output matrix and the first Lyapunov exponent are as follows:

$$
\left[\begin{array}{ccc}
x_{0} & \cdots & x_{n}+(m+1) \operatorname{tri}\left(q_{n}\right) y_{n} \\
y_{0} & \cdots & y_{n} \\
q_{0} & \cdots & q_{n}
\end{array}\right]
$$

$$
\begin{aligned}
& {\left[\begin{array}{ccc}
x_{0}+\varepsilon & \cdots & x_{n n}+(m+1) \operatorname{tri}\left(q_{n n}\right) y_{n n} \\
y_{0} & \cdots & y_{n n} \\
q_{0} & \cdots & q_{n n}
\end{array}\right]} \\
& L E_{1}^{m+1}=\lim _{\substack{n \rightarrow \infty \\
\varepsilon \rightarrow 0}} \frac{1}{n} \ln \left|S_{1}+(m+1) S_{2}\right|>0
\end{aligned}
$$

From the inequality (3-10), it is easy to obtain (I) $S_{1}$ and $S_{2}$ have the same positive and negative properties; (II)When $S_{1}$ and $S_{2}$ are different in positive and negative properties, $\left|S_{2}\right|>2\left|S_{1}\right|$.

(I)When the positive and negative properties of $S_{1}$ and $S_{2}$ are the same:

We can obtain from (3-11):

$\left|S_{1}+(m+1) S_{2}\right|>\left|S_{1}+m S_{2}\right|>1$

Proposition is proved.

(II)When the positive and negative properties of $S_{1}$ and $S_{2}$ are different, and $\left|S_{2}\right|>2\left|S_{1}\right|$ :

From (3-9), (3-10), $\left|S_{1}\right|>1, m \in \mathrm{N}^{*}$, we can obtain:

$\left|S_{1}+m S_{2}\right|>\left|(2 m-1) S_{1}\right|>1$

That is

$\left|S_{1}+(m+1) S_{2}\right|>\left|(2 m+1) S_{1}\right|>1$

From relation (II) and inequality (3-12), it can be seen that $S_{1}+S_{2}$ and $S_{2}$ have the same positivity and negativity, and $S_{1}+m S_{2}$ and $S_{2}$ also have the same positivity and negativity. The deduction can be obtained,

$\left|S_{1}+(m+1) S_{2}\right|-\left|S_{1}+m S_{2}\right|>0$

The proposition is proved.

In fact, as long as the parameter $a \neq 0$, a positive number $\xi$ can always be found, so that within the infinite wide parameter range of $[\xi, \infty]$, the system has at least one Lyapunov exponent that is always greater than 0 and gradually increases as $k$ increases, that is, the state of the new system can always remain a chaotic state or a hyperchaotic state within this infinitely wide parameter range. Similarly, the parameter also has an infinitely wide range of chaotic or hyperchaotic parameters, the proving process is similar to the above. 


\section{Super multi-stability phenomenon}

In a dissipative chaotic system, without changing the system parameters, the system presents a variety of phase trajectories of different positions or topological structures with the change of initial conditions, that is, the coexistence of multiple attractors. This phenomenon is also called multi-stability. Particularly, when the number of coexisting attractors tends to be infinite, this behavior is called the super multi-stability.

Let $a=10, k=10$, and $x_{0}$ and $y_{0}$ are 1 and change $q_{0}$. The phase diagram is shown in the Fig.8.

It can be seen from the Fig. 8 that when other conditions remain unchanged, $q_{0}=q_{00}+2 m$ is assigned, and $m$ is an arbitrary integer, an infinite number of coexisting attractors can be generated in the phase space.

In order to better analyze the super multi-stability of the system, $x_{0}$ and $q_{0}$ are taken as horizontal and vertical coordinates to describe the suction basin of the system, as shown in the Fig.9.

As shown in the Fig.9, the different color areas in the suction basin represent different attractors. The new system as a whole has an attraction area with a period of 2, indicating that the system has super multi-stability properties. At the same time, it should be noted that the junction of different suction areas is not smooth, which means that at the critical point, a small change of $x_{0}$ may also cause the transition of different attractive domains. When $q_{0}= \pm i$ and $i$ is any odd number, a periodic coexistence attractor appears in the system, but this phenomenon only appears when both $k$ and $a$ are integers.

4.1 Analysis of the emergence mechanism of super multi-stability

In fact, no matter what the values of $a$ and $k$ take, when only the initial value $q_{0}$ is changed, and at this time, let $q_{0}=q_{00}+2 m, q_{00}$ is any constant, and $m$ is any integer, the system can present the properties of an infinite number of attractors.

This phenomenon is very interesting, and the mechanism by which an infinite number of coexistence attractors are generated will be analyzed below.

When the initial value is $\left[x_{0}, y_{0}, q_{00}\right]$, the output will be $\left[x_{1}, y_{1}, q_{1}\right],\left[x_{2}, y_{2}, q_{2}\right], \ldots,\left[x_{n}, y_{n}, q_{n}\right]$ under the iteration of the difference equation.

Then, set the initial value as $\left[x_{0}, y_{0}, q_{00}+2 m\right]$, the output is:

$\left[x_{11}, y_{11}, q_{11}\right]=\left[x_{1}, y_{1}, q_{1}+2 m\right]$
Substitute $\left[x_{11}, y_{11}, q_{11}\right]$ as input into the iterative equation, the output is:

$\left[x_{22}, y_{22}, q_{22}\right]=\left[x_{2}, y_{2}, q_{2}+2 m\right]$

If the iteration is repeated, the output is always:

$\left[x_{n n}, y_{n n}, q_{n n}\right]=\left[x_{n}, y_{n}, q_{n}+2 m\right]$

Therefore, by changing the initial value $q_{0}$, an infinite number of coexisting attractors can be described in the phase space. At the same time, inspired by the mechanism of generating infinite multiple coexisting attractors in this system, this paper proposes a new method, which makes it easier for ordinary discrete chaotic systems by a slight modification to obtain super multistability. This paper takes the transformation of a 3D discrete chaotic system as an example, and it can expand the transformation of other $n \mathrm{D}$ systems. The method can be summarized as follows:

(1)Set one of the state variables in the differential equation as the input of the memristor (2-1) without loss of generality, and set $z_{n}$ as the input, then the memristor function is $M\left(z_{n}\right)=\operatorname{tri}\left(\pi z_{n}\right)$;

(2)Let the memristor be coupled with two other state variables. In particular, in the equations of $x_{n+1}$ and $y_{n+1}$, there should be no other item containing $z_{n}$ except as the input of the memristive function and the input with the same period as the memristive function;

(3)Let $z_{n+1}=z_{n}+f\left(x_{n}, y_{n}, z_{n}\right)$, and in the same way, the term containing $z_{n}$ in $f\left(x_{n}, y_{n}, z_{n}\right)$ can only appear as the input with the same period as the memristive function.

\subsection{Application of Super Multi Stability Mechanism}

Literature [23] proposed a new 3D discrete chaotic system

$$
\left\{\begin{array}{l}
x_{n+1}=-a-x_{n}+y_{n}+x_{n}^{2}-x_{n} y_{n}+y_{n}^{2} \\
y_{n+1}=b-x_{n}+x_{n} y_{n} \\
z_{n+1}=-x_{n} y_{n}-y_{n} z_{n}-x_{n} z_{n}
\end{array}\right.
$$

Among them, $a$ and $b$ are system parameters. When $a=0.32, b=3$, and the initial values are $[0.7,0.3,0.4]$, the Lyapunov exponent of the system is $(0.167,-0.410$, $-0.428)$, and the system is in a state of chaotic motion . The attractor phase diagram is shown in the Fig.10.

Using the above method to modify the system (4-1) to make its new kinetic equation as follows:

$\left\{\begin{array}{l}x_{n+1}=-a-x_{n}+y_{n}+x_{n}^{2}-x_{n} y_{n}+y_{n}^{2} \\ y_{n+1}=b-x_{n}+x_{n} y_{n}+k M\left(z_{n}\right) x_{n} \\ z_{n+1}=z_{n}-x_{n} y_{n}-y_{n}-x_{n}\end{array}\right.$ 


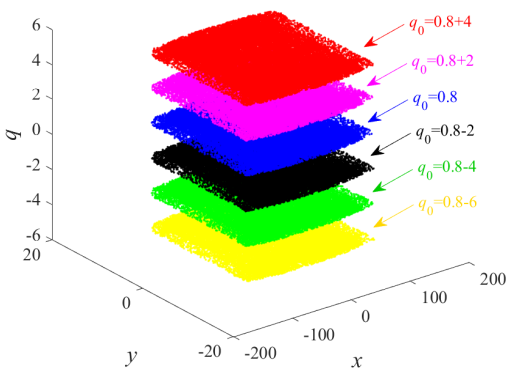

(a) Three-dimensional phase diagram

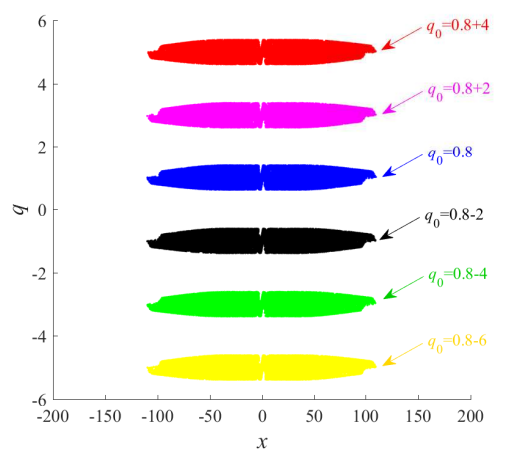

(b) $x-q$ plane phase diagram

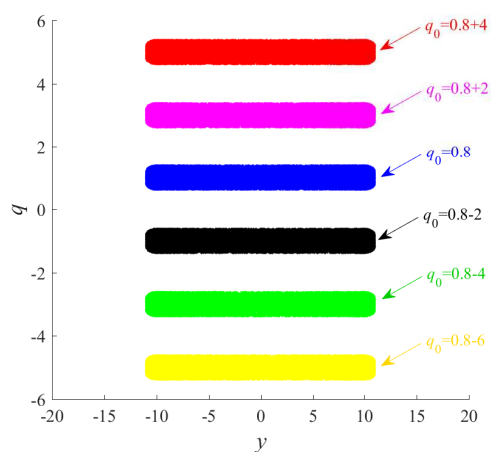

(c) $y-q$ plane phase diagram

Fig. 8: Phase diagram of coexistence attractor

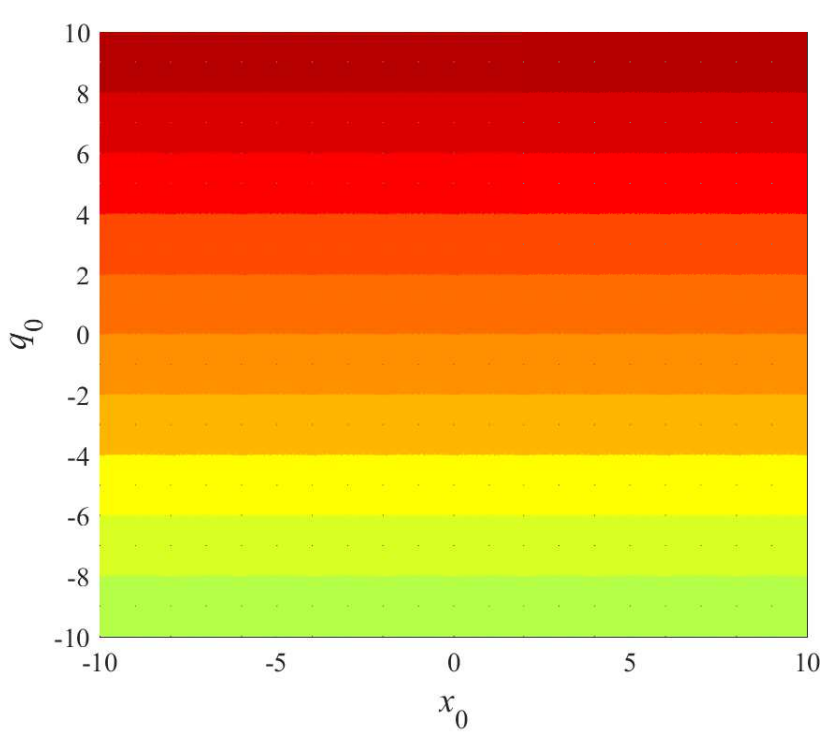

Fig. 9: The suction basin of the new system

Where $a, b$, and $k$ are the system control parameters. Set $a=-0.62, b=0.22, x_{0}=0.7, y_{0}=0.3$, and the initial value $z_{n}$ of $z_{0}$ as different values, the bifurcation diagram of the Lyapunov exponent spectrum and state variable $z_{n}$ can be shown in the Fig. 11 .

The third Lyapunov exponent is a small negative number, which is not shown in Fig.11.

Set the initial value as $[0.7,0.3,0.4]$, and by changing the values of $a, b, k$ and of the system (4-2), the different states can be obtained, as shown in Table 3 .

Keep other conditions remain unchanged, and change the initial value $z_{0}$, as shown in the Fig.12, the existence of the coexistence attractor of the system can be observed in the phase space.

From the Fig.11, 12 and Table 1, it is obvious that when using the above method to modify an ordinary $3 \mathrm{D}$ discrete chaotic system, the new system not only

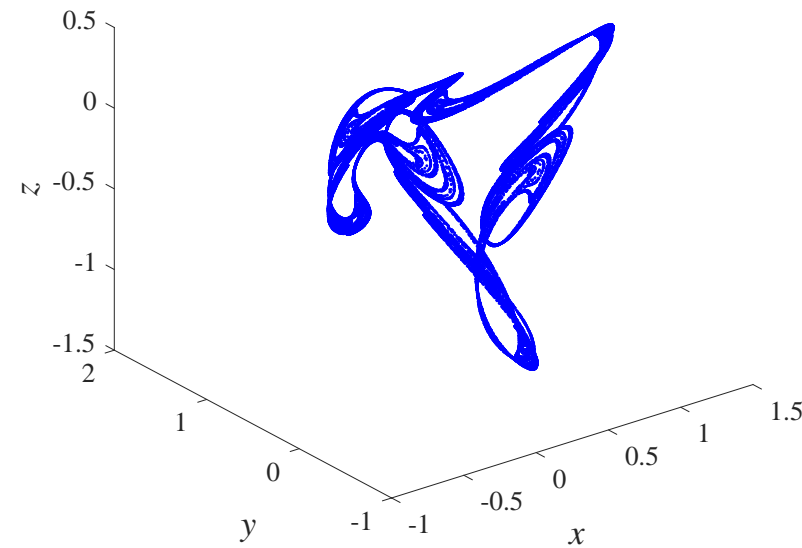

Fig. 10: Three-dimensional phase diagram of chaotic state of system (4-1)

Table 3: Several different values of system parameters and their corresponding Lyapunov exponents and state values

\begin{tabular}{lcc}
\hline \multicolumn{1}{c}{$a . b$ and $k$} & LEs & system status \\
\hline$-0.62,0.22,0.77$ & $-0.0418,-0.088,-2.068$ & Cycle 1 \\
$-0.62,0.16,0.9$ & $0.1372,-1.08,-2.068$ & Chaotic state \\
$-0.62,0.22,0.908$ & $0.0908,0.0436,-1.359$ & Hyperchaotic state \\
\hline
\end{tabular}

has super multi-stability properties, but also produces a hyperchaotic state that the original system does not have. Compared with the original system, the modified system has more complex dynamic behaviors. It is proved that this method is effective to modify the ordinary chaotic system. 

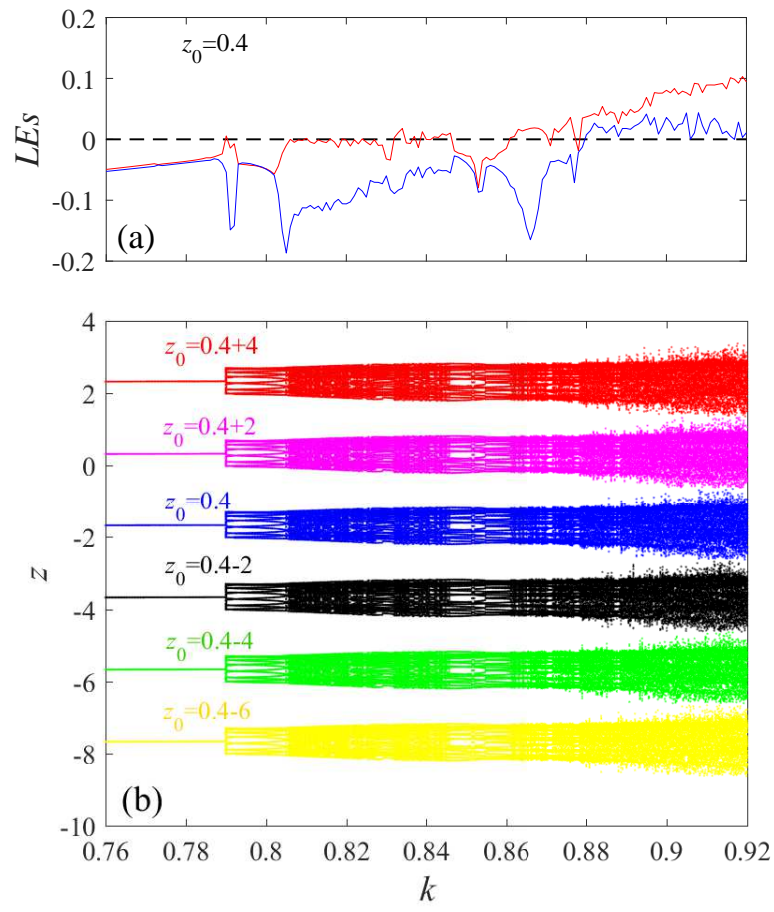

Fig. 11: System (4-2) Lyapunov exponent spectrum and bifurcation diagram

\section{Hardware platform implementation}

The key to the application of the chaotic system is the design of the hardware circuit. Because the system (31) has super multi-stability properties which depends on the initial conditions. In actual engineering, certain requirements are put forward for the stability and reliability of the hardware platform. The circuit composed of discrete electronic components has a complex structure and low accuracy, which is particularly sensitive to interference of the external environment, such as changes in temperature and humidity, while the DSP hardware platform is affected a little. Therefore, this paper uses the DSP hardware platform with chip model TMS320F28335 to implement the new system.

Set $k=0.35, a=0.14$, and the initial value is $[1,1,0.8]$. At this time, the system (3-1) is in a chaotic state. Observe the result with an oscilloscope, as shown in the Fig.13.

Keep other conditions remain unchanged and set the initial values of $q_{0}$ as 0.8 and 2.8 respectively. The results display on the oscilloscope as shown in the Fig.14.

Set $k=0.8, a=0.3$, and the initial value is $\left[1,1, q_{0}\right]$, the system (3-1) is in a hyperchaotic state. Similarly, change the value of $q_{0}$ to 0.8 and 2.8 respectively, as shown in the Fig.15.
Compared with the Fig.5, it is found that the chaotic signal generated based on The DSP hardware platform is consistent with the simulations.

\section{Conclusion}

In this paper, a discrete memristor model based on triangular wave function is designed, and a new $3 \mathrm{D}$ discrete memristive chaotic map with infinitely wide parameter range based on it is proposed. The Lyapunov exponent spectrum, bifurcation diagram, phase diagram, etc. are analyzed, which show that the new system can maintain the hyperchaotic characteristics within a large parameter range. Furthermore, an improved perturbation method is proposed to prove that the new system has an infinitely wide range of chaotic state parameter. At the same time, the maximum Lyapunov exponent of the system increases with the increase of the control parameters, and the dynamic behavior and ergodicity are enhanced. Next, the super multi stability property of the new system is analyzed, and the generation mechanism of this property is analyzed and summarized. Then, an ordinary 3D discrete chaotic map is modified to obtain super multi stability property, which proves that the modification method is effective. Finally, the new system is implemented by using the DSP hardware platform, which lays a foundation for its future applications in chaotic secure communication.

Acknowledgements This work was supported by the National Natural Science Foundation of China (No. 61203004), the Natural Science Foundation of Heilongjiang Province (No. F201220) and the Heilongjiang Province Natural Science Foundation Joint Guidance Project (No. LH2020F022).

\section{Data availability statement}

The datasets generated during and/or analyzed during the current study are available from the corresponding author on reasonable request.

\section{Conflict of interest}

The authors declare that they have no conflict of interest.

\section{References}

1. X. Wang, Y. Yu, J. Cai, N. Yang, K. Shi, S. Zhong, K. Adu, N. Tashi, Multiple mismatched synchronization for coupled 


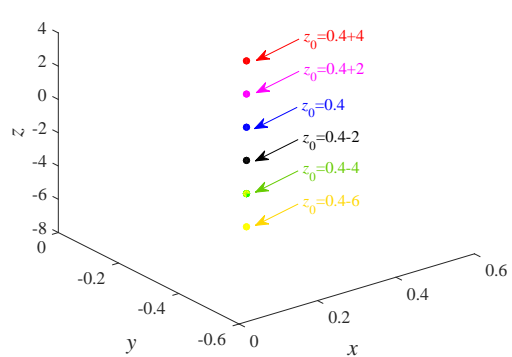

(a) Cycle 1 coexistence attractor

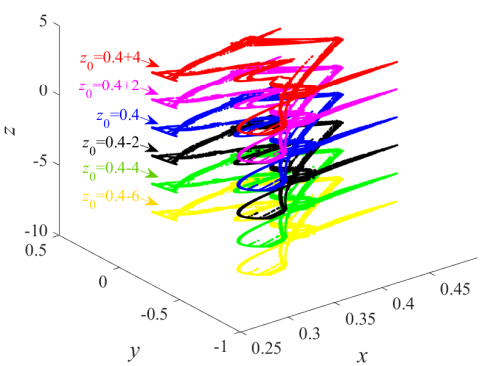

(b) Chaotic state coexistence attractor

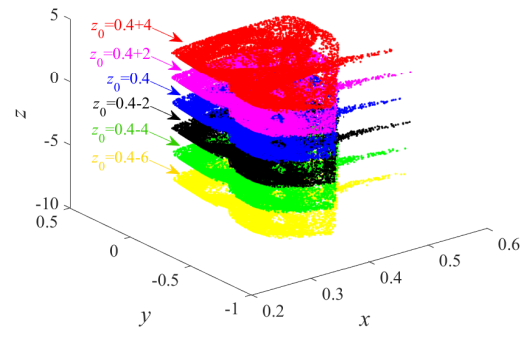

(c) Hyperchaotic coexistence attractor

Fig. 12: Coexistence attractor of system (4-2)

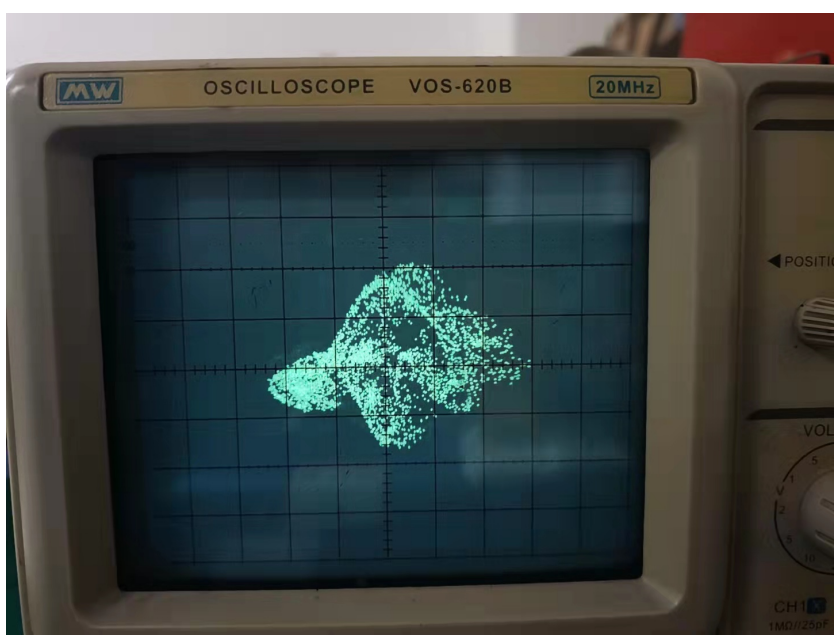

(a) $x-y$ plane phase diagram

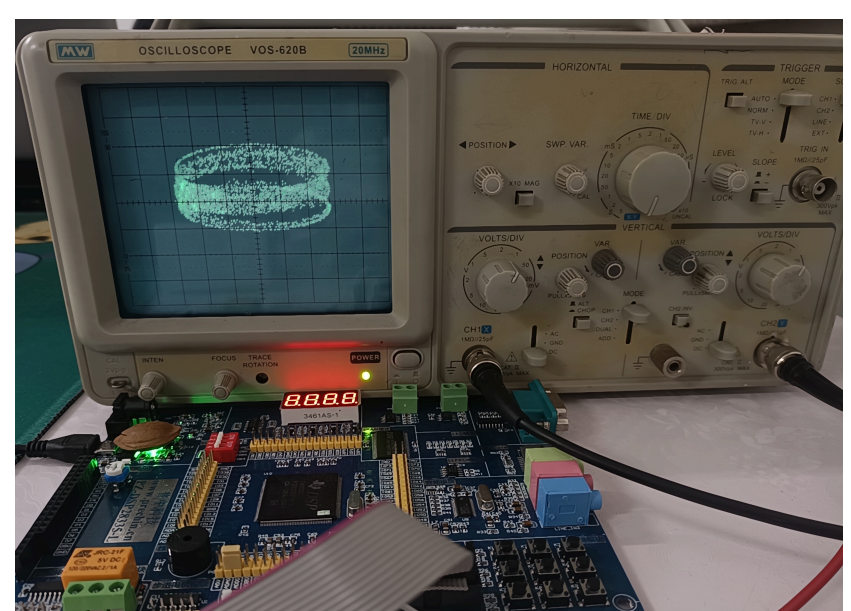

(b) $y-q$ plane phase diagram

Fig. 13: The physical object of the hardware platform and the chaotic attractor produced by it

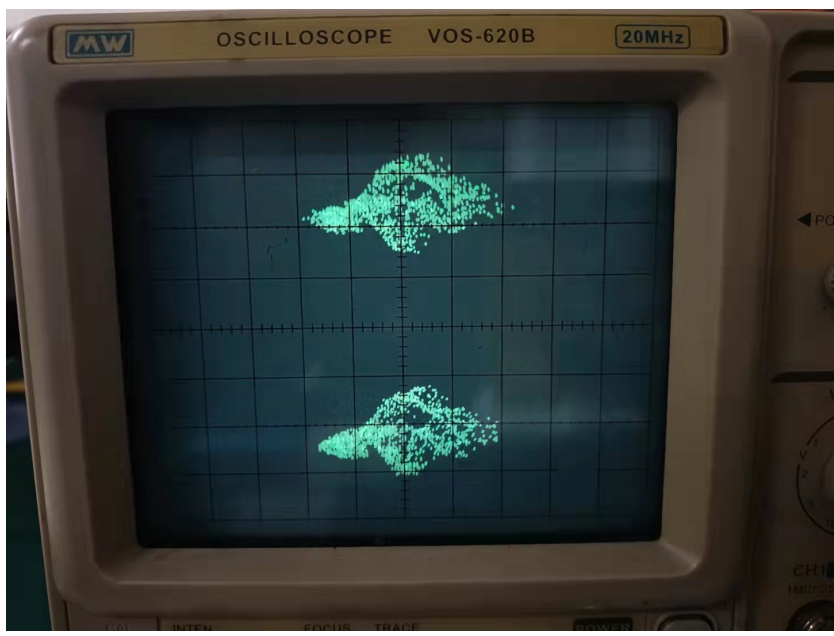

(a) The $x-q$ plane coexistence attractor phase diagram

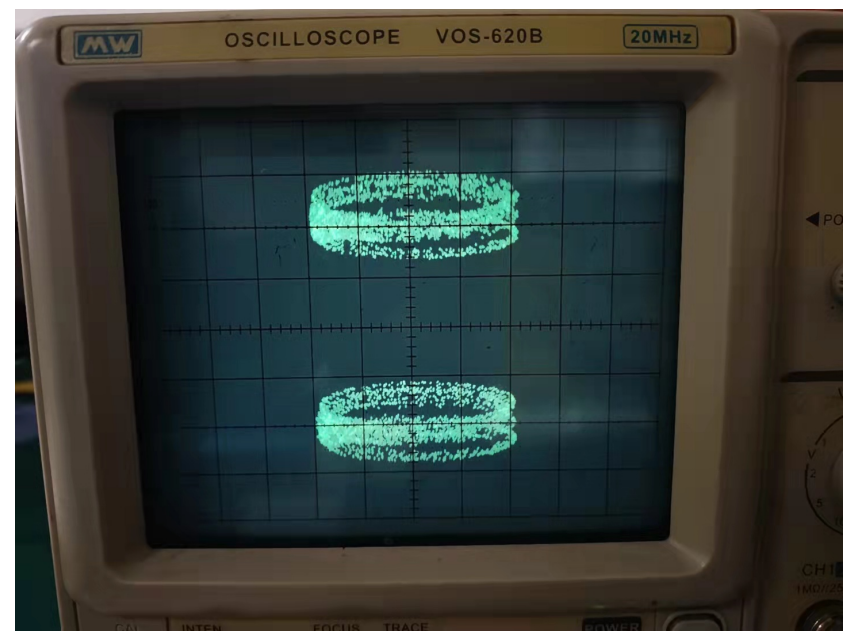

(b) The $y-q$ plane coexistence attractor phase diagram

Fig. 14: Coexistent chaotic attractor 


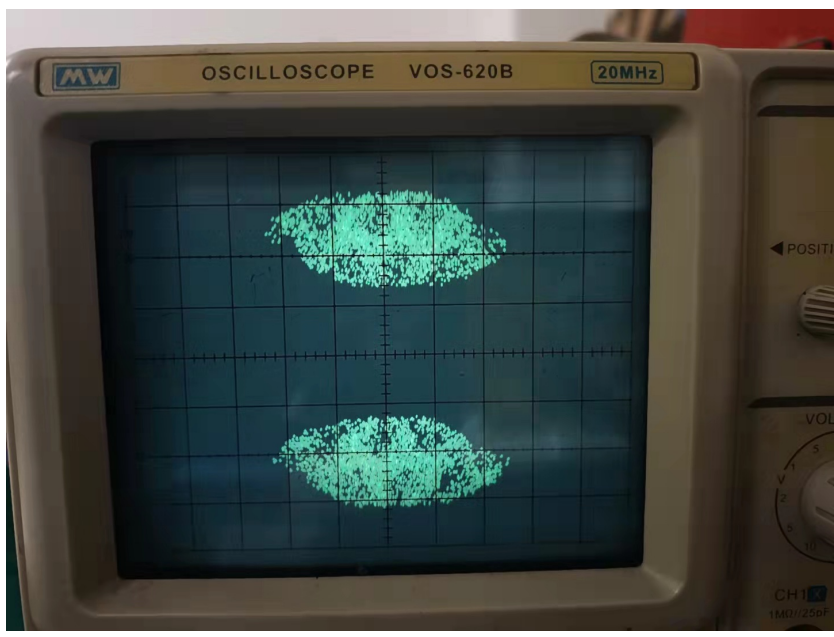

(a) The $x-q$ plane coexistence attractor phase diagram

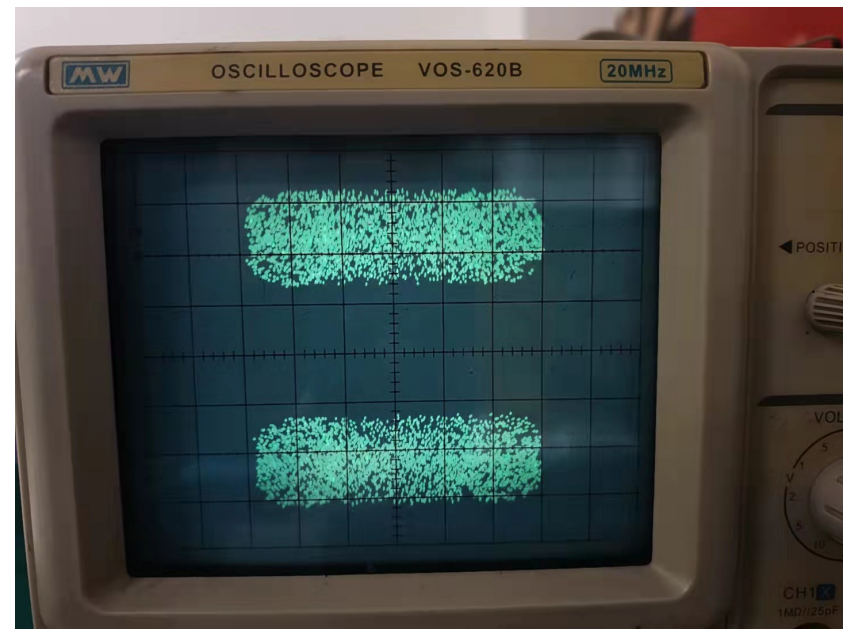

(b) The $y-q$ plane coexistence attractor phase diagram

Fig. 15: Hyperchaotic coexistence attractor

memristive neural networks with topology-based probability impulsive mechanism on time scales, IEEE Transactions on Cybernetics (2021).

2. Z. Yan, X. Huang, J. Liang, Aperiodic sampled-data control for stabilization of memristive neural networks with actuator saturation: A dynamic partitioning method, IEEE Transactions on Cybernetics (2021).

3. M. J. Aljafar, M. A. Perkowski, J. M. Acken, R. Tan, A time-efficient cmos-memristive programmable circuit realizing logic functions in generalized and-xor structures, IEEE Transactions on Very Large Scale Integration (VLSI) Systems (2018) 1-14.

4. G. Pooja, S. Murali Krishna, V. Ravi, Multi-level memristor memory: Design and performance analysis, Int. J. Innov. Technol. Explor. Eng 8 (2019) 723-729.

5. M. Chen, M. Sun, H. Bao, Y. Hu, B. Bao, Flux-charge analysis of two-memristor-based chua's circuit: Dimensionality decreasing model for detecting extreme multistability, IEEE Transactions on Industrial Electronics (2019) 1-1.

6. D.-W. Ding., F.-F. Liu., H. Chen., N. Wang., D. Liang., Sliding mode control of fractional-order delayed memristive chaotic system with uncertainty and disturbance, Communications in Theoretical Physics 68 (12) (2017) 741.

7. X. Zhang, C. Li, Y. Chen, I. U. Herbert, T. Lei, A memristive chaotic oscillator with controllable amplitude and frequency, Chaos Solitons \& Fractals 139 (2020) 110000.

8. Z. J. Li, Y. C. Zeng, A memristor oscillator based on a twin-t network, Chinese Physics B (4) (2013) 6.

9. Njitacke, Z., T., Kengne, J., Tapche, R., Wafo, Pelap, F., Uncertain destination dynamics of a novel memristive $4 \mathrm{~d}$ autonomous system, Chaos, Solitons and Fractals: Applications in Science and Engineering: An Interdisciplinary Journal of Nonlinear Science 107 (2018) 177-185.

10. Y. Fang, D. Yue, Y. Li, G. Wang, The amplitude, frequency and parameter space boosting in a memristormeminductor-based circuit, Nonlinear Dynamics 96 (1) (2019).

11. B. Aala, C. Ncb, B. Qwa, Ergodic sensitivity analysis of one-dimensional chaotic maps, Theoretical \& Applied Mechanics Letters 10 (6) (2020) 10.

12. Z. Wei, R. Wang, A. Liu, A new finding of the existence of hidden hyperchaotic attractors with no equilibria, Mathematics and Computers in Simulation 100 (2014) 13-23.
13. C. Chen, K. Sun, S. He, A class of higher-dimensional hyperchaotic maps, The European Physical Journal Plus 134 (8) (2019) 1-13.

14. H. Li, Z. Hua, H. Bao, L. Zhu, B. Bao, Two-dimensional memristive hyperchaotic maps and application in secure communication, IEEE Transactions on Industrial Electronics (2020).

15. J. Liu, W. Zhang, A new three-dimensional chaotic system with wide range of parameters, Optik - International Journal for Light and Electron Optics 124 (22) (2013) 55285532 .

16. X. Changbiao., Z. De., X. Cheng., L. Zhou, A new chaotic system with parameter $\mathrm{b}$ in a super-large range and its adaptive sliding mode control, JOURNAL OF VIBRATION AND SHOCK 38 (3) (2019) 6.

17. Z. Wei., W. Zhou-qing, Analysis and fpga implementation of a novel super-wide range hyperchaotic system, MICROELFCTRONICS \& COMPUTER 36 (8) (2019) 5.

18. X. Changbiao., Z. De., G. Taotao., A large range chaotic system with multiple types of equilibrium points and its topological horseshoe, JOURNAL OF VIBRATION AND SHOCK 39 (9) (2020) 8.

19. Z. Ze-Feng., H. Li-Lian., X. Jian-Hong., L. Shuai., Dynamic study of a new five-dimensional conservative hyperchaotic system with wide parameter range, Acta Physica Sinica 40 (5) (2021) 14

20. D. B. Strukov, H. Kohlstedt, Resistive switching phenomena in thin films: Materials, devices, and applications, Mrs Bulletin 37 (02) (2012) 108-117.

21. S. Kvatinsky, G. Satat, N. Wald, E. G. Friedman, U. C. Weiser, Memristor-based material implication (imply) logic: Design principles and methodologies, IEEE Transactions on Very Large Scale Integration (VLSI) Systems 22 (10) (2014) 2054-2066.

22. Y. Simin., L. Jinhu., L. Chengqing., Some progresses of chaotic cipher and its applications in multimedia secure communications, Journal of Electronics \& Information Technology 38 (3) (2016) 18.

23. L. De-kui, W. Xing-min, Dynamics and chaos control of a new three-dimensional discrete system, Journal of Liaoning University(Natural Science Edition) 48 (1) (2021) 6 . 